\title{
Static properties and semileptonic decays of doubly heavy baryons in a nonrelativistic quark model
}

\author{
C. Albertus ${ }^{1, a}$, E. Hernández ${ }^{2, b}$, J. Nieves $^{1}$, and J.M. Verde-Velasco ${ }^{2}$ \\ 1 Departamento de Física Atómica, Molecular y Nuclear, Universidad de Granada, E-18071 Granada, Spain \\ 2 Grupo de Física Nuclear, Departamento de Física Fundamental e IUFFyM, Facultad de Ciencias, E-37008 Salamanca, Spain \\ Original article: Eur. Phys. J. A 32, 183 (2007) DOI: 10.1140/epja/i2007-10364-y
}

Received: 7 March 2008

Published online: 14 March 2008 - C Società Italiana di Fisica / Springer-Verlag 2008

We have noticed that we missed a symmetry factor $1 / \sqrt{2}$ for baryons with two equal heavy quarks that affects our results in sect. 5. As a consequence the factor of 2 in the r.h.s. of eqs. (30) and (31) should read $\sqrt{2}$ instead. This induces corrections in form factors and decay widths:

- The numerical values for all form factors displayed in fig. 5 should be divided by a factor $\sqrt{2}$.

- The factor of 2 in the r.h.s. of eqs. (36) and (38) should be $\sqrt{2}$. The statement before eq. (36) ("On the other hand... have") is not correct and it should read as follows: "On the other hand, if the two quarks in the current had the same flavour, vector current conservation would fix the normalization of the zeroth component of the vector current matrix element at $w=1$, since this just counts the number of heavy quarks, two, in that case. For quarks with distinct flavours, but still in the limit $m_{b}=m_{c}$, the forward vector matrix element has an extra Clebsch-Gordan factor of $1 / \sqrt{2}$, and thus we have"

- The r.h.s. of eqs. (B.2)-(B.5) should be divided by a factor $\sqrt{2}$.

- The numerical values for all differential decay widths shown in fig. 6 should be divided by a factor 2 . The same factor of 2 affects our results for decay widths shown in tables 8 and 9 .

Decay width results calculated in ref. [41] for $b b \rightarrow b c$ and $b c \rightarrow c c$ transitions and in ref. [44] for the $\Xi_{b c} \rightarrow \Xi_{c c}$ transition suffer also from the use of incorrect symmetry factors, as confirmed to us by the authors of those works (D. Ebert, R.N. Faustov, V.O. Galkin, private communication; M.A. Sanchis-Lozano, private communication). The numerical values taken from these references and shown in our table 9 should be corrected by a factor $1 / 2$ and 2 , respectively. Note that in these circumstances our results continue to be in reasonable agreement with those of ref. [41], while the result in ref. [44] is now much larger.

Concerning also table 9, one cannot compare directly our result for the $\Xi_{b c} \rightarrow \Xi_{c c}$ transition with the one in ref. [42]. The reason being that in the latter the $\Xi_{b c}$ state is defined with the $c$ and light quark spins coupled to total spin 1. Had they coupled the $b c$ pair to spin 1, they would have obtained a decay width value which would have been roughly 4 times larger, and thus in agreement with our corrected results (Th. Gutsche, private communication).

We have to correct also our statement in footnote 12. Private discussions with the authors of ref. [41] confirm that their calculation preserves vector current conservation in the equal mass case. Our incorrect statement about their calculation of $h_{-}(\omega)$ was based in their approximate eqs. (36) and (40)-(41) which seem not to be valid in the equal mass case.

Besides, we noticed the following misprints:

- In table 8 the upper error on $\Gamma_{L}$ for the $\Xi_{b c} \rightarrow \Xi_{c c}$ transition should have been 0.22 and not 0 . as it appeared. On top of that one would have to correct it by the factor of $1 / 2$ mentioned above.

- In table 9 , the result quoted 28.8 from ref. [43] $\left(\Omega_{b b} \rightarrow \Omega_{b c}\right.$ transition) should be omitted.

a Present address: School of Physics and Astronomy, University of Southampton, Southampton SO17 1BJ, UK.

b e-mail: gajatee@usal.es 